\title{
STIGMA RECEPTIVITY TEST IN DIVERSE SPECIES OF TOMATO
}

\section{MONIKA A. MAKWANA ${ }^{1} \&$ PARIHAR AKARSH ${ }^{2}$}

${ }^{1}$ Department of Genetics and Plant Breeding, B. A. College of Agriculture, Anand Agricultural University, Anand, Gujarat, India

${ }^{2}$ Department of Agricultural biotechnology, Anand Agricultural University, Anand, Gujarat, India

\section{ABSTRACT}

The present experiment on tomato comprised of 17 genotypes belonging to ten different species. The research was carried out at "Research Centre for Distant Hybridization in Field and Fruit Crops, Dept. of Agricultural Biotechnology, Anand Agricultural University, Anand during 2016-2017'. Stigma receptivity being one of the important traits influencing the rate of pollination and successful fertilization was under taken in the present study. The observations were recorded from tomato species on stigma receptivity by using Hydrogen peroxide. The stigma was found receptive before 12 hrs. of an thesis and may continue upto withering stage of flower, which was the common pattern observed in all the species. Stigma became fully receptive after opening of flower bud. But, in case of accession LA 1777 (S. habrochaites), the stigma did not become receptive at bud stage, while stigma of LA 2157 (S. arcanum) and LA 2819 (S. lycopersicum) showed receptivity from bud stage.

KEYWORDS: Anthesis, Stigma, Hydrogen Peroxide S. habrochaites, S. arcanum \& S. lycopersicum

Received: Aug 01, 2017; Accepted: Aug 14, 2017; Published: Aug 18, 2017; Paper Id.: IJASROCT20171

\section{INTRODUCTION}

The tomato belongs to the family Solanaceae (also known as the nightshade family), genus Solanum, subfamily Solanoideae and tribe Solaneae. Tomato (Solanum lycopersicum) is a major fruit crop with great economic importance worldwide that is used for both fresh consumption and processing. It provides many benefits to human nutrition and health. In addition, tomato has become a model plant in a wide range of research disciplines, including genetics (Chetelat et al., 2000; Tanksley, 2004), evolution (Peralta et al., 2005; Moyle, 2008), and reproductive biology.

Presently, there are 16 wild species of tomato including S. habrochaites, S. pennellii, S. pimpinellifolium, S. cheesmaniae, S. galapagense, S. peruvianum, S. corneliomulleri, S. chilense, S. chmielewskii, S. arcanum, S. neorickii, S. huaylasense, S. lycopersicoides, S. ochranthum, S. jugandifolium and S. sitiens (Knapp et al., 2003; Bedinger et al., 2011). These all species in the tomato clade are diploid with the same chromosome number $(2 \mathrm{n}=$ $2 \mathrm{x}=24$ ) and are considered to have evolved primarily by genic changes rather than large-scale chromosomal rearrangements (Anderson et al., 2010).

Stigma receptivity is a crucial stage in the maturation of a flower which may greatly influence the rate of self-pollination, pollination success at different stages in the flower life cycle, the relative importance of various pollinators, the interference between male and female functions, the rate of competition via improper pollen transfer, and the chances of gametophytic selection (Galen et al., 1987). The information about duration of stigma receptivity may be very well utilized for making crossing or hybridizing program successful. Receptive stigmas 
are characterized by high enzymatic activity. The presence of several enzymes is found to coincide with this developmental stage (Knox, 1984; Shivana and Rangaswamy, 1992) and consequently most of the methods to determine stigma receptivity in vitro are based on the identification of enzymatic activity (Knox et al., 1986; Dafni 1992; Kearns and Inouye, 1993). This paper presents a known method for stigma receptivity using hydrogen peroxide (6\%) in tomato.

\section{MATERIALS AND METHODS}

Stigma receptivity was studied by hydrogen peroxide test under microscope in a laboratory. The flowers were taken at three stages viz, before a thesis (bud stage), full bloom and withering stage. The stigmas from flowers were kept on cavity slide then hydrogen peroxide (6\%) was dropped on it. It was observed under zeiss microscope at $10 \mathrm{X}$ and $40 \mathrm{X}$ microscopic fields. The bubbling from stigma was considered as mark of receptivity of stigma. The observations were recorded in 1 hour interval from 8 a.m. to 8 p.m.

\section{RESULTS AND DISCUSSIONS}

The pistils were long and thin in wild species and the stigmas were exposed on the outside of the anther cone. The light green stigmas seemed somewhat swollen and were difficult to distinguish from the connecting styles. In contrast, the stubby pistils of cultivated genotypes were entirely embedded within the anther cone and the conspicuous capitates stigma was distinct from the style.

The observation regarding this trait was taken from accessions of all species taken under this experiment, which are given in Table 1.1 and Figure, 2-3. A solution of hydrogen peroxide (6\%) was placed on the stigma and the appearance of bubbles was observed under microscope and scored. The test was carried out in diverse tomato species at discrete classes of age viz. (A) bud stage, (B) freshly opened stage and (C) withering stage to determine period and duration of stigma receptivity. The reactivity was scored based on the extent of bubbling. From the present study, it was observed that in all the species, stigma became receptive one day before opening of flower and remained receptive up to withering stage. However, in some species viz., LA 2157 (S. arcanum), LA 3859 (S. pimpinellifolium), LA 0490 (S. lycopersicum), LA 2817 (S. lycopersicum), LA 2819 (S. lycopersicum), LA 4440 (S. lycopersicum) and GT-2 (S. lycopersicum) the bubbling was also found higher in withering stage.

In contrast, at bud stage, very low reactivity was scored by LA 1777 (S. habrochaites) while LA 2157 (S. arcanum) and LA 2819 (S. lycopersicum) scored moderate and high reactivity, respectively. The above results revealed that in LA 1777 (S. habrochaites) the stigma did not become receptive at bud stage while stigma of LA 2157 (S. arcanum) and LA 2819 (S. lycopersicum) showed receptivity from bud stage. The receptivity of stigma increased gradually in bud stage as after $5.00 \mathrm{pm}$ i.e. before $12 \mathrm{hrs}$. Of anthesis, which was the common pattern observed in all the species under study. Stigma became fully receptive after opening of flower bud.

Stigma of older flowers (C) which tested positive for peroxidase activity showed a varying level of reaction. LA 3652 (S. lycopersicum var. cerasiforme), WIR 13708 (S. lycopersicum var. cerasiforme), LA 2556 (S. hirsutum), EC52005 (S. hirsutum) and EC-520047 (S. chilense) showed lower bubbling while WIR 5032 (S. chilense) and EC-520047 (S. chmielewskii) showed lesser extent of bubbling. In some accessions viz., LA 2157 (S. arcanum), LA 3859 (S. chilense), LA 0490 (S. lycopersicum), LA 2817 (S. lycopersicum), LA 2819 (S. lycopersicum), LA 4440 (S. lycopersicum) and GT-2 (S. lycopersicum), the reactivity was found higher in $\mathrm{C}$ stage as observed in the B stage of flower in other genotypes. 
The higher activity of hydrogen peroxide, suggested that the $\mathrm{H}_{2} \mathrm{O}_{2}$ is predominant in stigma. The $\mathrm{H}_{2} \mathrm{O}_{2}$ or reactive oxygen species (ROS) found in most of the plants as well as animals which play a diverse role in cell signaling, where they regulate diverse aspects of plant metabolism and cell growth. The $\mathrm{H}_{2} \mathrm{O}_{2}$ found to be involved in hypersensitive response during pathogen attack and programmed cell death (Bolwell, 1996; Blee et al., 2001; Bolwell et al., 2002). At withering stage, the cells might have gone through programmed cell death leading to accumulation of $\mathrm{H}_{2} \mathrm{O}_{2} /$ ROS which showed more bubbling.

Sidhu et al. (1980), Kalloo (1991) and Kaul (1991) studied stigma receptivity in tomato and reported that in tomato, stigma becomes receptive 16 to 18 hours before anthesis and retains the receptivity upto six days after anthesis.

Further, the reliability of peroxidase test was supported by Dupui \& Dumas, 1990 and Mc Inni et al., 2006, who reported that, the receptive stigmas 'ripe' for pollination and are characterized by high levels of peroxidase activity and these tests are widely used to determine pistil receptivity measuring stigma peroxidase activity (Dafni \& Maues, 1998).

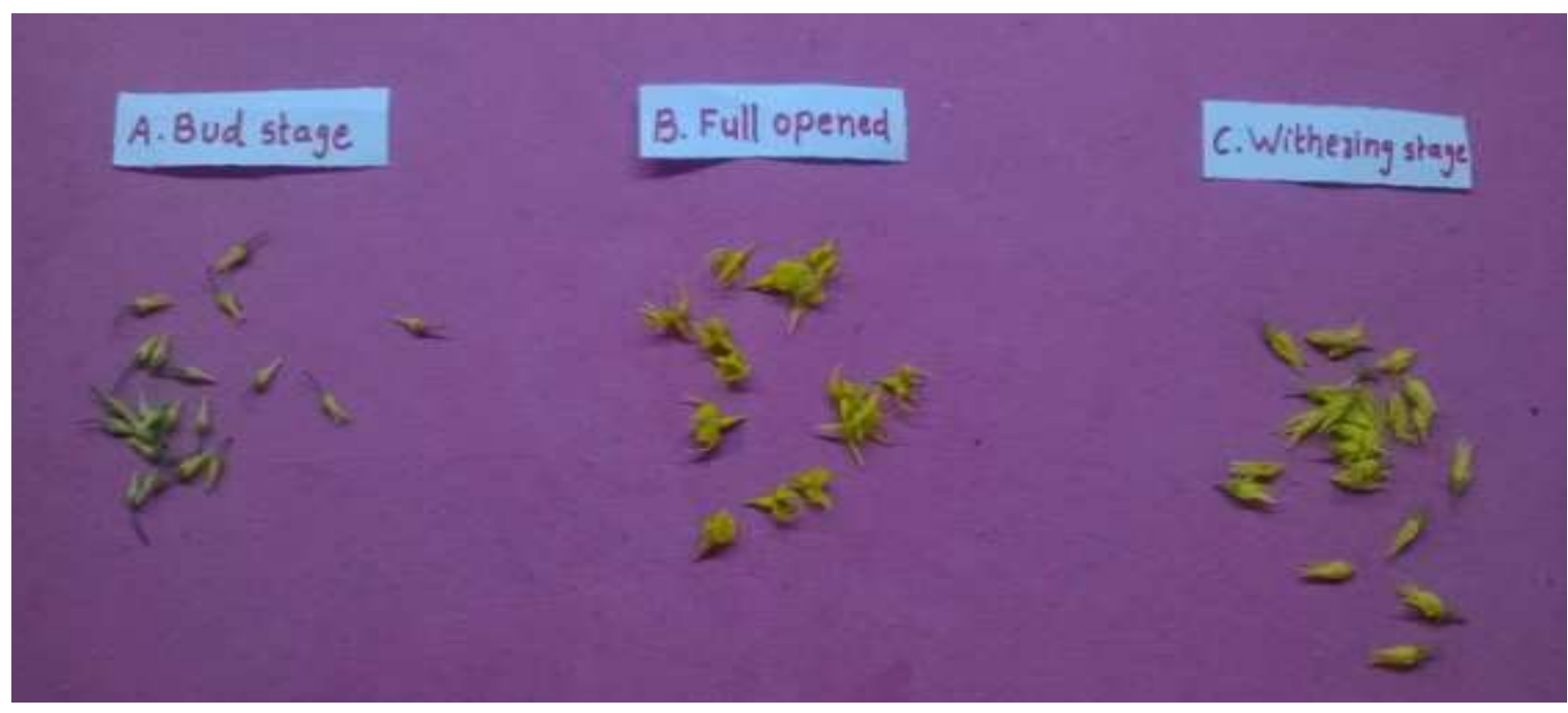

Figure 1: Stages of Flowers Collected for Stigma Receptivity 
Table 1.1 Stigma Receptivity by Hydrogen Peroxide $\left(\mathrm{H}_{2} \mathbf{o}_{2}\right)$ in Various Cultivated and Wild Species / Accessions of Tomato

\begin{tabular}{|c|c|c|c|c|c|c|c|c|c|c|c|c|c|c|c|}
\hline $\begin{array}{l}\text { Sr. } \\
\text { No. }\end{array}$ & Genotype & $\begin{array}{l}\text { Species/ } \\
\text { Genotypes }\end{array}$ & Stage & $\begin{array}{l}\text { 8am- } \\
9 \mathrm{am}\end{array}$ & $\begin{array}{l}\text { 9am- } \\
\text { 10am }\end{array}$ & $\begin{array}{l}\text { 10am- } \\
11 \mathrm{am}\end{array}$ & $\begin{array}{l}11 \mathrm{am}- \\
12 \mathrm{am}\end{array}$ & $\begin{array}{l}\text { 12noon } \\
1 \mathrm{pm}\end{array}$ & $\begin{array}{l}1 \mathrm{pm}- \\
2 \mathrm{pm}\end{array}$ & $\begin{array}{l}2 \mathrm{pm}- \\
3 \mathrm{pm}\end{array}$ & $\begin{array}{l}3 \mathrm{pm}- \\
4 \mathrm{pm}\end{array}$ & $\begin{array}{l}4 \mathrm{pm}- \\
5 \mathrm{pm}\end{array}$ & $\begin{array}{l}5 \mathrm{pm}- \\
6 \mathrm{pm}\end{array}$ & $\begin{array}{l}6 \mathrm{pm}- \\
7 \mathrm{pm}\end{array}$ & $\begin{array}{l}7 \mathrm{pm}- \\
8 \mathrm{pm}\end{array}$ \\
\hline \multirow[t]{3}{*}{1} & \multirow[t]{3}{*}{ LA 3652} & \multirow{3}{*}{$\begin{array}{l}\text { S. } \\
\text { lycopersicum } \\
\text { var. } \\
\text { cerasiforme }\end{array}$} & $\mathrm{A}$ & - & - & - & - & + & + & + & + & ++ & ++ & ++ & +++ \\
\hline & & & B & ++ & +++ & ++++ & ++++ & ++++ & ++++ & ++++ & ++++ & ++++ & ++++ & ++++ & ++++ \\
\hline & & & C & - & + & + & ++ & ++ & + & + & + & + & + & + & + \\
\hline \multirow[t]{3}{*}{2} & \multirow{3}{*}{$\begin{array}{l}\text { WIR } \\
13708\end{array}$} & \multirow{3}{*}{$\begin{array}{l}\text { S. lycopersicum } \\
\text { var. } \\
\text { cerasiforme }\end{array}$} & $\mathrm{A}$ & + & + & + & - & + & - & + & + & - & - & ++ & ++ \\
\hline & & & $\mathrm{B}$ & ++ & ++ & ++ & +++ & ++++ & ++++ & ++++ & +++ & +++ & +++ & ++++ & ++++ \\
\hline & & & $\mathrm{C}$ & + & + & - & - & - & + & + & + & + & + & ++ & - \\
\hline \multirow[t]{3}{*}{3} & \multirow[t]{3}{*}{ LA 2556} & \multirow[t]{3}{*}{ S. hirsutum } & $\mathrm{A}$ & + & + & - & - & - & + & ++ & +++ & + & +++ & +++ & +++ \\
\hline & & & B & ++ & ++ & ++ & +++ & ++++ & ++++ & ++++ & ++++ & ++++ & +++++ & ++++ & ++++ \\
\hline & & & $\mathrm{C}$ & + & + & + & + & ++ & + & + & + & + & + & + & + \\
\hline \multirow[t]{3}{*}{4} & \multirow{3}{*}{$\begin{array}{l}\text { EC- } \\
520058\end{array}$} & \multirow[t]{3}{*}{ S. hirsutum } & A & + & + & + & + & + & ++ & ++ & ++ & ++ & +++ & +++ & +++ \\
\hline & & & $\mathrm{B}$ & +++ & +++ & +++ & +++ & ++++ & ++++ & +++++ & +++++ & ++++ & ++++ & +++ & ++++ \\
\hline & & & $\mathrm{C}$ & + & + & + & ++ & + & + & + & + & + & + & + & - \\
\hline \multirow[t]{3}{*}{5} & \multirow[t]{3}{*}{ EC-52004 } & \multirow[t]{3}{*}{ S. cheesmaniae } & $\mathrm{A}$ & + & + & + & + & + & + & + & + & + & ++ & +++ & +++ \\
\hline & & & $\mathrm{B}$ & ++ & ++ & +++ & +++ & ++++ & ++++ & ++++ & ++++ & ++++ & ++++ & ++++ & ++++ \\
\hline & & & $\mathrm{C}$ & ++ & + & + & + & + & + & + & + & + & + & + & + \\
\hline 6 & WIR & S. chilense & $\mathrm{A}$ & + & - & + & + & ++ & - & +++ & +++ & ++ & ++ & +++ & +++ \\
\hline & 5032 & & B & ++ & ++ & ++ & ++ & +++ & ++++ & ++++ & ++++ & +++++ & +++++ & ++++ & +++ \\
\hline & & & $\mathrm{C}$ & ++ & ++ & ++ & ++ & ++ & ++ & + & + & +++ & ++ & +++ & +++ \\
\hline 7 & EC- & S. chmielewskii & $\mathrm{A}$ & + & + & + & + & + & + & + & + & + & + & + & + \\
\hline & 520047 & & $\mathrm{~B}$ & ++ & ++ & +++ & +++ & ++++ & ++++ & ++++ & ++++ & ++++ & ++++ & ++++ & ++++ \\
\hline & & & $\mathrm{C}$ & +++ & + & ++ & ++ & ++ & + & +++ & +++ & +++ & ++ & +++ & +++ \\
\hline 8 & IIHR & S. peruvianum & A & - & - & - & - & - & - & - & - & - & + & ++ & +++ \\
\hline & 1970 & & B & ++++ & ++++ & ++++ & ++++ & ++++ & ++++ & +++++ & +++++ & ++++ & ++++ & ++++ & ++++ \\
\hline & & & $\mathrm{C}$ & +++ & +++ & ++ & ++ & +++ & ++ & +++ & +++ & +++ & ++ & ++ & ++ \\
\hline 9 & LA 1777 & S. habrochaites & $\mathrm{A}$ & + & + & - & - & - & + & + & + & ++ & ++ & +++ & +++ \\
\hline & & & $\mathrm{B}$ & ++++ & ++++ & ++++ & +++++ & +++++ & +++++ & +++++ & +++++ & +++++ & +++++ & ++++ & ++++ \\
\hline & & & $\mathrm{C}$ & +++ & +++ & +++ & ++ & + & +++ & - & - & ++ & +++ & - & - \\
\hline 10 & LA 2779 & S. chilense & $\mathrm{A}$ & + & + & - & + & + & ++ & ++ & ++ & + & ++ & ++ & ++ \\
\hline & & & $\mathrm{B}$ & +++ & ++++ & ++++ & ++++ & ++++ & +++ & ++++ & ++++ & +++ & ++++ & ++++ & ++++ \\
\hline & & & $\mathrm{C}$ & +++ & +++ & +++ & + & +++ & - & +++ & +++ & ++ & ++ & - & - \\
\hline 11 & LA 2157 & S. arcanum & $\mathrm{A}$ & + & + & - & - & ++ & ++ & ++ & ++ & ++ & +++ & +++ & +++ \\
\hline & & & B & ++++ & +++ & ++++ & +++++ & ++++ & ++++ & +++++ & +++++ & +++++ & +++++ & +++++ & +++++ \\
\hline & & & $\mathrm{C}$ & ++++ & ++ & + & ++ & +++ & +++ & ++ & ++ & +++ & +++ & +++ & +++ \\
\hline 12 & LA 3859 & $S$ & $\mathrm{~A}$ & - & + & + & ++ & + & ++ & +++ & +++ & ++ & +++ & +++ & +++ \\
\hline & & pimpinellifolium & B & +++ & +++ & +++++ & ++++ & ++++ & ++++ & ++++ & ++++ & ++++ & +++++ & +++++ & +++++ \\
\hline & & & $\mathrm{C}$ & ++ & - & + & +++ & +++ & +++ & $++t$ & $++t$ & ++ & ++ & ++ & ++ \\
\hline 13 & LA 0490 & S. lycopersicum & $\mathrm{A}$ & + & ++ & + & + & + & + & ++ & ++ & ++ & +++ & +++ & +++ \\
\hline & & & $\mathrm{B}$ & ++ & ++++ & ++++ & ++++ & +++++ & +++++ & +++++ & +++++ & +++++ & +++++ & ++++ & +++++ \\
\hline & & & $\mathrm{C}$ & +++ & ++++ & ++++ & ++++ & ++++ & +++ & +++ & +++ & +++ & ++++ & +++ & +++ \\
\hline 14 & LA 2817 & S. lycopersicum & $\mathrm{A}$ & + & + & + & + & + & + & +++ & +++ & +++ & +++ & ++++ & ++++ \\
\hline & & & B & ++ & ++++ & ++++ & +++ & +++++ & +++++ & ++++ & ++++ & ++++ & ++++ & ++++ & +++++ \\
\hline & & & $\mathrm{C}$ & +++ & ++++ & ++++ & +++ & +++ & +++ & +++ & +++ & +++ & +++ & +++++ & +++++ \\
\hline 15 & LA 2819 & S. lycopersicum & $\mathrm{A}$ & + & ++ & +++ & +++ & +++ & ++ & ++++ & ++++ & ++ & ++++ & ++++ & ++++ \\
\hline & & & $\mathrm{B}$ & ++++ & +++++ & +++++ & +++++ & +++++ & +++++ & ++++ & ++++ & ++++ & +++++ & +++++ & +++++ \\
\hline & & & C & +++ & ++++ & ++ & ++++ & ++++ & +++++ & +++ & +++ & +++ & ++++ & +++ & +++++ \\
\hline 16 & LA 4440 & S. lycopersicum & $\mathrm{A}$ & - & - & - & + & ++ & - & + & + & ++ & ++ & ++ & +++ \\
\hline & & & B & ++++ & ++++ & +++++ & ++++ & ++++ & +++++ & +++ & +++ & ++++ & ++++ & +++ & ++++ \\
\hline & & & $\mathrm{C}$ & +++ & +++ & +++ & ++++ & +++++ & ++++ & ++++ & ++++ & ++ & +++ & ++++ & ++++ \\
\hline 17 & GT-2 & S. lycopersicum & $\mathrm{A}$ & - & + & ++ & + & ++ & + & ++ & ++ & + & ++ & +++ & +++ \\
\hline & & & $\mathrm{B}$ & +++ & ++++ & ++++ & +++++ & +++++ & +++++ & +++++ & +++++ & +++++ & +++++ & +++++ & ++++ \\
\hline & & & $\mathrm{C}$ & ++ & ++ & ++ & +++ & +++++ & ++++ & +++ & +++ & ++++ & ++++ & +++ & ++ \\
\hline
\end{tabular}

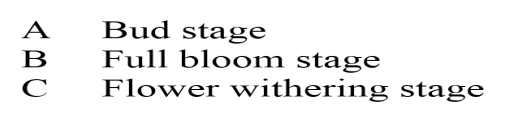

$\begin{array}{ll}- & \text { No reactivity } \\ + & \text { Very less reactivity } \\ ++ & \text { Less reactivity } \\ +++ & \text { Moderate reactivity } \\ ++++ & \text { High reactivity } \\ +++++ & \text { Very high reactivity }\end{array}$



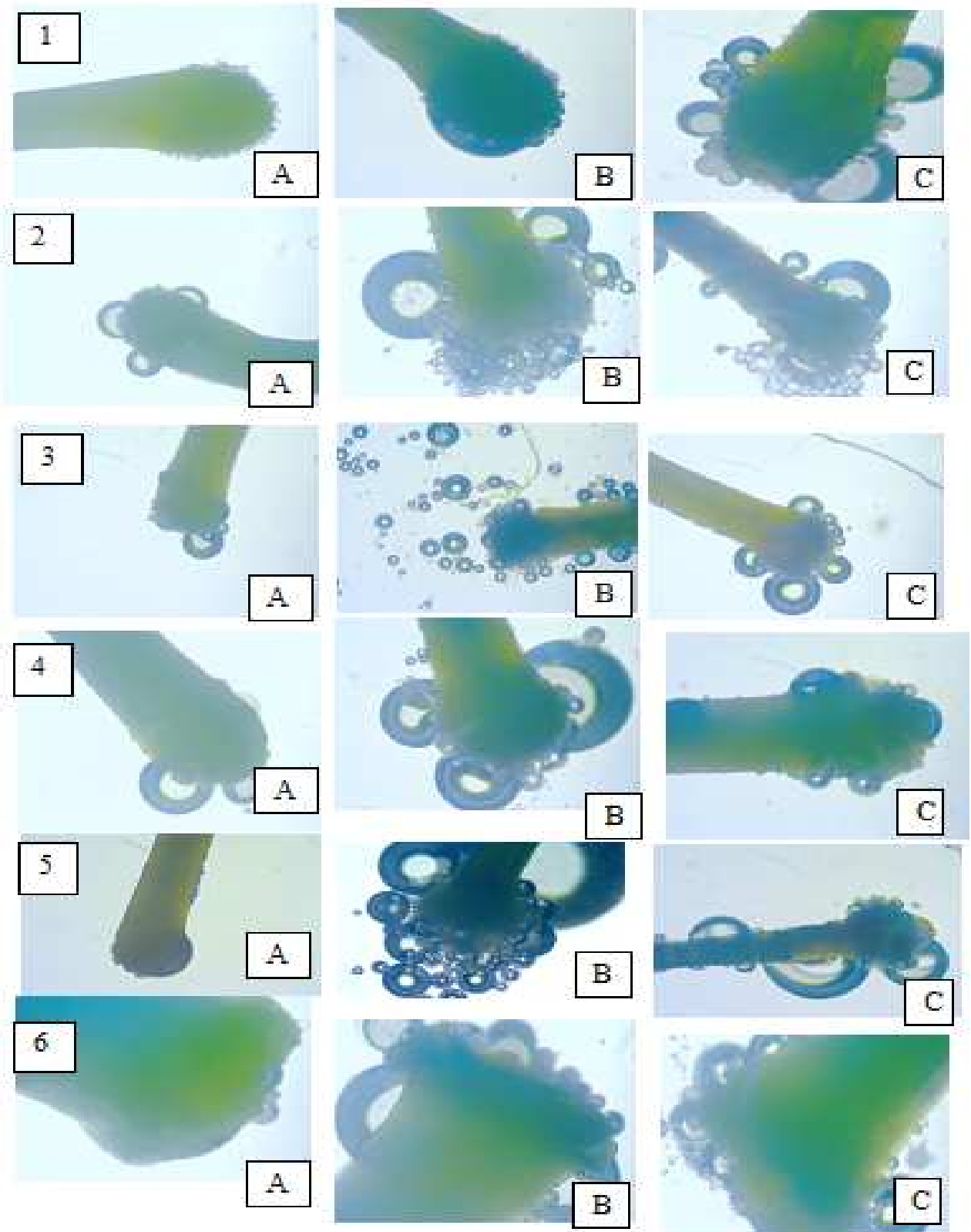

Figure 2: Stigma Receptivity using Peroxidase Test at Various Stages of Flower (1-6) A= Bud Stage, B= Full Bloom Stage, $C=$ Flower Withering Stage

1. LA 1777 (S. habrochaites), 2. LA 1970 (S. peruvianum), 3. WIR 5032 (S. chilense), 4. EC-520047 (S. chilense), 5. LA 2157 (S. arcanum), 6. LA 0490 (S. lycopersicum), 7. LA 4440 (S. lycopersicum), 8. GT-2 (S. lycopersicum), 9. LA 3652 (S. lycopersicum var. cerasiforme), 10. LA 2556 (S. hirsutum), 11. EC-52004 (S. cheesmaniae) 

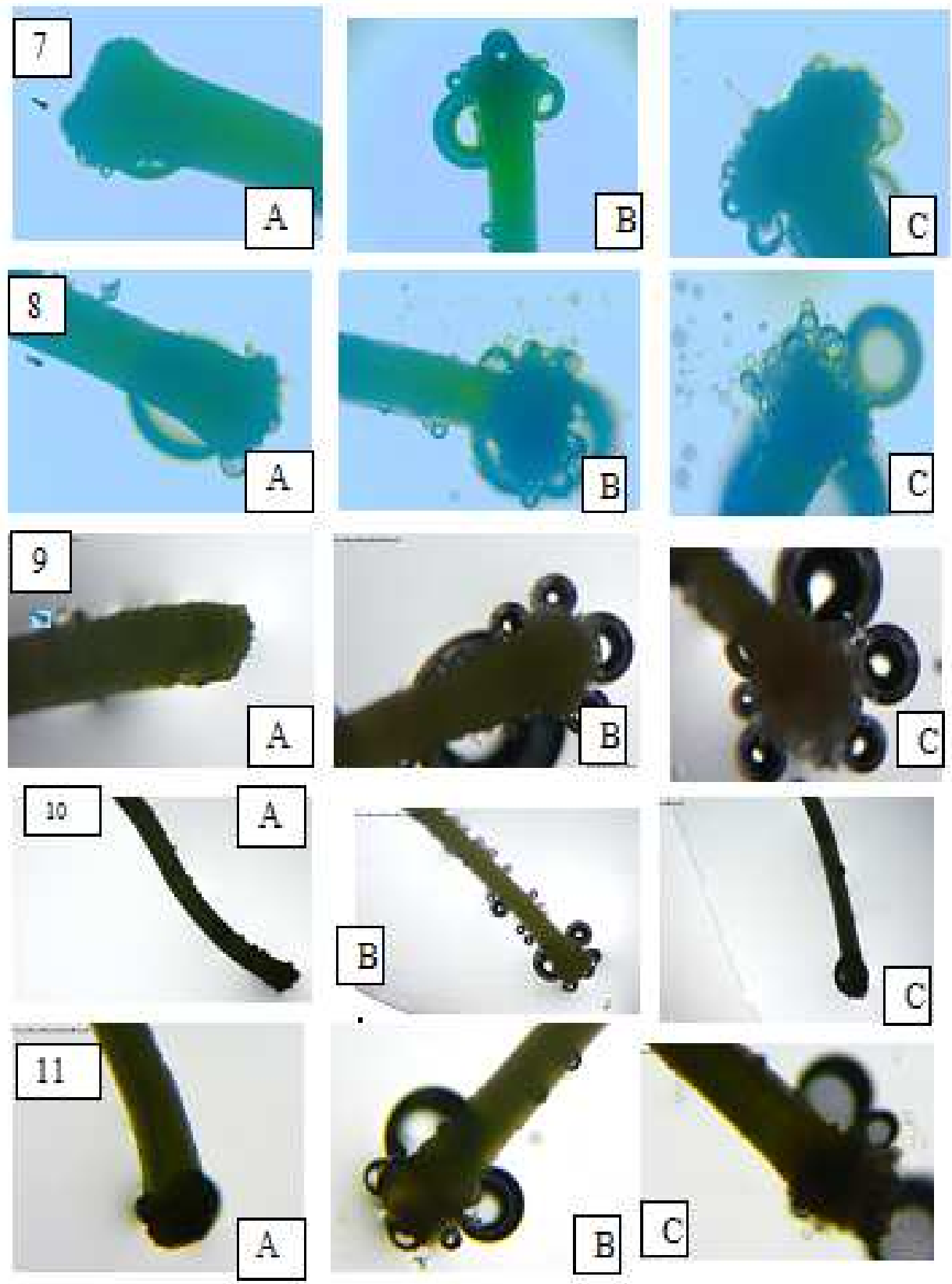

Figure 3: Stigma Receptivity using Peroxidase Test at Various Stages of Flower (7-11) A= Bud Stage, B= Full Bloom Stage, $C=$ Flower Withering Stage

1. LA 1777 (S. habrochaites), 2. LA 1970 (S. peruvianum), 3. WIR 5032 (S. chilense), 4. EC-520047 (S. chilense), 5. LA 2157 (S. arcanum), 6. LA 0490 (S. lycopersicum), 7. LA 4440 (S. lycopersicum), 8. GT-2 (S. lycopersicum), 9. LA 3652 (S. lycopersicum var. cerasiforme), 10. LA 2556 (S. hirsutum), 11. EC-52004 (S. cheesmaniae)

\section{REFERENCES}

1. Anderson, L. K., Covey, P. A., Larsen, L. R., Bedinger, P. A. \& Stack, S. M. (2010). Structural differences in chromosomes distinguish species in the tomato clade. Journal of Cytogen Genome Resources, 2, 1-14.

2. Bedinger, P. A., Chetelat, R. T., McClure, B., Moyle, L. C., Rose, J. K., Stack, S. M., Van der Knaap, E., Baek, Y. S., LopezCasado, G., Covey, P. A., Kumar, A., Li, W., Nunez, R., Cruz-Garcia, F. and Royer, S. (2011). Interspecific reproductive 
barriers in the tomato clade: opportunities to decipher mechanisms of reproductive isolation. Sex. Plant Reprod., 24, 171-187.

3. Chetelat, R. T., Meglic, V. and Cisneros, P. (2000). A genetic map of tomato based on BC(1) Lycopersicon esculentum, Solanum lycopersicoides reveals overall synteny but suppressed recombination between these homeologous genomes. Genetics, 154: 857-867.

4. Dafni, A. \& Maues, M. M. (1998). A rapid and simple procedure to determine stigma receptivity. Sexual Plant Reproduction, 11, 177-180.

5. Dafni, A. (1992). Pollination ecology: a practical approach. Oriole Press at Oxford University Press, Oxford.

6. Dupuis, I. \& Dumas, C. (1990). Biochemical markers of female receptivity in maize (Zea mays L.) assessed using in vitro fertilization. Plant Science, 70, 11-20.

7. Galen, C., Zimmer, K. A. \& Newport, M. E. (1987). Pollination and floral scent morphs of Polemonium viscosum: a mechanism for disruptive selection on flower size. Evolution, 41:599-606.

8. Kalloo, G. (1991). In genetic improvement of tomato, Berlin: Springer-Verlag.

9. Kaul (1991). Genetic improvement and reproductive biology in tomato. Monographs on theoretical and applied genetics, 3950 .

10. Kearns, A. C. and Inouye, D. W. (1993). Techniques for pollination biologists. University of Colorado Press.

11. Knapp, S., Darwin, S. C. \& Pelatra, I. E. (2003). Taxonomy of tomatoes in the Galápagos Islands, Native and introduced species of Solanum section Lycopersicon (Solanaceae). Syst. Biodiversity, 12, $29-53$.

12. Knox, R. B. (1984). Pollen-pistil interaction. In: Linskens HF, Heslop- Harrison J (eds) Cellular interactions. Encyclopedia of Plant Physiology, vol. 17. Springer, Berlin Heidelberg New York.

13. Knox, R. B., Williams, E. G. and Dumas, C. (1986). Pollen, pistil and reproductive function in crop plants. Plant Breed Rev, 4 , 9-79.

14. Mc Innis, S. M., Emery, D. C., Porter, R., Desikan, R., Hancock, J. T. \& Hiscock, S. J. (2006). The role of stigma peroxidases in flowering plants: insights from further characterization of a stigma-specific peroxidase (SSP) from Senecio squalidus (Asteraceae). Journal of Experimental Botany, 57, 1835-1846.

15. Moyle, L. C. and Nakazato, T. (2008). Comparative genetics of hybrid incompatibility: sterility in two Solanum species crosses. Genetics, 179, 1437-1453.

16. Peralta, Iris E. \& Spooner, David M. (2005). New species of wild tomatoes (Solanum section Lycopersicum, Solanaceae) from Northern Peru. Systematic Botany, 30, 424-434.

17. Shivana, K.R. and Rangaswamy, N. S. (1992). Pollen biology: a laboratory manual. Springer, Berlin.

18. Sidhu, A. S., Kallo, G \& Pandita, M. C. (1980). Studies on some important aspects of floral biology in vegetable crops. Haryana J. Horti. Sci., 9, 207-217.

19. Tanksley, S. D. 2004. The genetic, developmental, and molecular bases of fruit size and shape variation in tomato. Plant Cell, 16,181-189. 
